\title{
SAÚDE DE MULHERES NO CLIMATÉRIO EM SISTEMA PRISIONAL
}

\author{
Rita de Cássia Ferreira dos Santos ${ }^{1}$, Audrey Vidal Pereira ${ }^{2}$, Valdecyr Herdy Alves ${ }^{3}$, Márcia Vieira dos Santo ${ }^{4}$, \\ Diego Pereira Rodrigues ${ }^{5}$, Giovanna Rosário Soanno Marchiori ${ }^{6}$
}

\begin{abstract}
RESUMO: Objetivou identificar sinais, sintomas e problemas que afetam a saúde, nos relatos de mulheres que passam pelo período do climatério, quando se encontram privadas de liberdade. Pesquisa exploratória, descritiva, com abordagem qualitativa. Entre outubro de 2014 e janeiro de 2015 foram realizadas entrevistas com sete mulheres que se encontravam no período do climatério, ao estarem em um presídio feminino situado no Estado do Rio de Janeiro, Brasil. A partir da análise temática, foram identificadas duas categorias: Sintomatologia referente ao período do climatério e Problemas que afetam a saúde de mulheres privadas de liberdade. A maioria das entrevistadas passa pelo período do climatério de maneira desconfortável, tendo a saúde afetada de algum modo durante a prisão. Assim, o climatério, sobretudo em situações de privação de liberdade, deve ser mais explorado pelas políticas públicas de saúde contribuindo para melhoria das condições de vida desse grupo específico.
\end{abstract}

DESCRITORES: Mulheres; Prisões; Saúde da mulher; Climatério; Enfermagem.

\section{HEALTH STATUS OF CLIMACTERIC WOMEN IN THE PRISON SYSTEM}

\begin{abstract}
The present study aimed to identify, based on reports, signs, symptoms and problems that affect the health of women in the climacteric period who are serving time in prison. Exploratory and descriptive study with a qualitative approach. Seven climacteric women incarcerated in a female prison in the State of Rio de Janeiro, Brazil, were interviewed from October 2014 to January 2015. After thematic analysis, two categories were identified: Symptoms related to the climacteric period and Problems that affect the health of women deprived of their liberty. Most interviewees reported feeling uncomfortable in the climacteric period, and having health problems in prison. Thus, climacteric, especially in situations of deprivation of liberty, should deserve greater attention of public health policies, to contribute to the improvement of the living conditions of this specific group.
\end{abstract}

DESCRIPTORS: Women; Prisons; Women's health; Climacteric; Nursing.

\section{SALUD DE MUJERES EN SISTEMA PENITENCIARIO DURANTE EL CLIMATERIO}

RESUMEN: Estudio cuyo objetivo fue identificar señales, síntomas y problemas que atingen la salud en los relatos de mujeres en el periodo del climaterio, cuando están privadas de libertad. Investigación exploratoria, descriptiva, de abordaje cualitativo. Las entrevistas fueron realizadas de octubre de 2014 a enero de 2015 con siete mujeres en el periodo del climaterio que estaban en una cárcel ubicada en el estado de Rio de Janeiro, Brasil. Por medio del análisis temático, se identificaron dos categorías: Sintomatología referente al periodo del climaterio y Problemas que atingen la salud de mujeres privadas de libertad. La mayoría de las entrevistadas siente malestar en el periodo de climaterio, teniendo la salud atingida de algun modo durante la cárcel. Así, el climaterio, sobretodo en situaciones de privación de libertad, debe ser más explorado por las políticas públicas de salud, contribuyendo para mejorar las condiciones de vida de ese grupo específico.

DESCRIPTORES: Mujeres; Prisiones; Salud de la mujer; Climaterio; Enfermería.

${ }^{1}$ Enfermeira. Universidade Federal Fluminense. Rio de Janeiro, RJ, Brasil.

${ }^{2}$ Enfermeiro. Doutor em Saúde Pública. Docente da Escola de Enfermagem Aurora de Afonso Costa, Universidade Federal Fluminense. Rio de Janeiro, RJ, Brasil.

${ }^{3}$ Enfermeiro. Doutor em Enfermagem. Docente da Escola de Enfermagem Aurora de Afonso Costa, Universidade Federal Fluminense. Rio de Janeiro, RJ, Brasil.

${ }^{4}$ Enfermeira. Mestre em Saúde Materno Infantil. Enfermeira do Sistema Penitenciário do Estado do Rio de Janeiro. Rio de Janeiro, RJ, Brasil.

${ }^{5}$ Enfermeiro. Mestre em Enfermagem. Docente do Centro Universitário Anhanguera de Niterói. Rio de Janeiro, RJ, Brasil.

${ }^{6}$ Enfermeira. Mestre em Saúde Materno Infantil. Docente da Faculdade Novo Milênio. Espírito Santo, ES, Brasil. 


\section{- INTRODUÇÃO}

As mulheres sofrem alterações físicas e psíquicas que afetam a saúde desde a adolescência até a terceira idade. Ao longo da vida são constantes as mudanças emocionais, físicas e sociais que interferem de modo significativo no processo de saúde e modos de vida das pessoas. Devido às mudanças no perfil demográfico mundial e brasileiro, têm sido crescentes as pesquisas focalizadas à mulher idosa tendo em vista esse conjunto de alterações ${ }^{(1)}$.

No Brasil, o envelhecimento populacional mostra tendência a uma feminização. Levando-se em conta o censo demográfico de 2010, do total da população brasileira (190.755.199 milhões de pessoas), 97 milhões são de mulheres (51\%) e 93 milhões (49\%) são de homens ${ }^{(1)}$.

Estima-se que o quantitativo de idosos, representado em 2000 por aproximadamente 15 milhões de pessoas vivendo com 60 anos ou mais de idade, possa exceder 30 milhões em 2020, totalizando por volta de $13 \%$ da população brasileira, e em 2060 a população de idoso passará para 33,7\%, e segundo o Instituto Brasileiro Geografia e Estatística (IBGE), o grupo de idosos em 2030 será maior que o grupo de crianças de até 14 anos de idade ${ }^{(2)}$. Diante do progressivo aumento da expectativa de vida feminina desde a segunda metade do século $\mathrm{XX}$, ocorre o aumento do número de mulheres que vivem $\mathrm{o}$ suficiente para chegar ao período de climatério ${ }^{(3)}$.

Segundo a Organização Mundial da Saúde (OMS), o climatério é definido como uma fase biológica da vida, que transita entre o período reprodutivo e não reprodutivo, descaracterizando a visão de que seja um processo patológico. Tem na menopausa um marco divisor assinalado pelo último ciclo menstrual, após 12 meses de sua ocorrência ${ }^{(4-6)}$.

As modificações presentes no período do climatério são influenciadas por diversos fatores, que variam de alterações nos níveis hormonais individuais até a forma como essas mulheres vivenciam essa etapa da vida. Desse modo, além das questões fisiológicas, torna-se necessário observar também os aspectos sociais e cultuais, as condições de vida e baixa hormonal, que interferem no período do climatério. Ou seja, é importante também atentar para fatores como: hábitos alimentares, atividade física, lazer, postura diante da vida, sexualidade, renda, inserção profissional e religiosa, dentre outros $^{(4-6)}$.

Estratégias de atenção à saúde durante o período do climatério devem ser incorporadas pelo Sistema Único de Saúde (SUS), em suas variadas especificidades, referentes aos diversos grupos femininos. Pois, mulheres com transtornos mentais, moradoras de rua, profissionais do sexo, ou as confinadas em instituições como hospitais psiquiátricos, conventos e presídios, podem sofrer discriminação e preconceito, convivendo com determinadas questões ou problemas de saúde geradores de maior vulnerabilidade ${ }^{(6)}$.

Ao encontro dessa proposta, desde 2014 no Brasil existe a Política Nacional de Atenção Integral à Saúde das Pessoas Privadas de Liberdade no Sistema Prisional (PNAISP), que busca dialogar com as diretrizes do $S U S^{(7)}$. De modo mais específico, com relação à saúde das mulheres privadas de liberdade foi elaborada a Política Nacional de Atenção às Mulheres em Situação de Privação de Liberdade e Egressa do Sistema Prisional ${ }^{(8)}$.

Assim, mediante características específicas do sistema prisional como o isolamento, superlotação, invasão de privacidade, vulnerabilidade, disseminação de doenças e situações de violência( ${ }^{(9-10)}$, entende-se que seja importante a atenção diferenciada relacionada às necessidades de saúde desse grupo específico - mulher que passa pelo período do climatério quando privada de liberdade.

Tendo em vista os inúmeros problemas que afetam a saúde das mulheres privadas de liberdade, sobretudo período do climatério, torna-se válido destacar os seguintes questionamentos: Quais os sinais e sintomas comuns ao período do climatério referidos por mulheres privadas de liberdade? Quais são os problemas de saúde referidos por estas mulheres durante o período do climatério dentro de uma penitenciária?

Dessa maneira, esta pesquisa teve por objetivo identificar sinais, sintomas e problemas que afetam a saúde, através dos relatos de mulheres que passam pelo período do climatério, quando se encontram privadas de liberdade. 
Trata-se de pesquisa descritiva, exploratória com abordagem qualitativa, que tem por intenção incorporar a questão do significado e da intencionalidade, buscando realizar menos generalizações e mais o aprofundamento e a compreensão do discurso dos sujeitos investigados ${ }^{(11)}$.

Esse estudo fez parte de um projeto temático, intitulado "Saúde da mulher em uma Penitenciária do Estado do Rio de Janeiro", do Grupo de Pesquisa: Maternidade: saúde da Mulher e da Criança, realizado com 40 mulheres selecionadas de modo aleatório a partir de uma lista nominal oficial da instituição pesquisada. Ocorreu no período de outubro de 2014 a janeiro de 2015, numa penitenciária gerenciada pela Secretaria de Administração Penitenciária do Estado do Rio de Janeiro, exclusiva para mulheres privadas de liberdade.

De acordo com a Resolução n466, de 12 de dezembro de 2012 do Conselho Nacional de Saúde $(\mathrm{CNS})^{(12)}$, que dispõe sobre pesquisa envolvendo seres humanos, o estudo foi aprovado em junho de 2014 pelo Comitê de Ética e Pesquisa da faculdade de medicina da Universidade Federal Fluminense (UFF), por meio do parecer Nº66.795/14 e pela Secretaria de Administração Penitenciária do Rio de Janeiro (SEAP-RJ), pelo processo E-2108753/2014.

Vale destacar que a assinatura do Termo de Consentimento Livre e Esclarecido (TCLE) não era permitida pela SEAP-RJ, pois a mesma não disponibilizava informações nominais das pessoas que se encontravam presas na instituição e nem autorizava o registro que identificasse essas informações em documentos que não fossem institucionais. A SEAP é o órgão que em nome do Estado, autoriza e se responsabiliza pela participação das(os) presidiárias(os) em atividades de pesquisas.

Do total das 40 participantes do projeto temático, compuseram a amostra deste estudo sete mulheres que estavam passando pelo período do climatério, com idade maior do que 35 anos e que aceitaram participar da investigação.

Foram considerados como critérios de inclusão: mulheres que referiram estar passando pelo período do climatério, com ou sem tratamento medicamentoso específico para menopausa ou climatério, e que se encontravam presas por mais de seis meses. E como critério de exclusão: mulheres privadas de liberdade num período menor que seis meses, e que se encontravam impossibilitadas de participar da pesquisa, diante da não autorização por algum motivo dos agentes penitenciários.

A participação das mulheres se deu de modo voluntário, com entrevistas semiestruturadas que foram realizadas de modo individual e reservado em ambiente determinado pela autoridade presente na instituição prisional. Antes do início das entrevistas, cada mulher era esclarecida sobre os objetivos da realização do estudo.

Como não era autorizada a utilização de recursos digitais pela SEAP em momentos de realização das entrevistas, as respostas foram transcritas de modo concomitante pela pesquisadora. Ao término das respostas, era solicitada para fosse realizada leitura de cada registro a fim de comprovar de acordo por parte da mulher. Mesmo que não tenham sido permitidas assinaturas do TCLE, foram garantidos processos de anonimato, sendo preservados o sigilo das identidades a partir de código alfanumérico (M1 a M7).

Para direcionar a realização das entrevistas, com vistas a identificar os sinais, os sintomas e os problemas relacionados à saúde de mulheres privadas de liberdade que estavam passando pelo período do climatério, foi utilizado roteiro semi-estruturado com questões relacionadas especificamente ao objeto de investigação.

Para análise dos resultados, utilizou-se a análise temática ${ }^{(13)}$, que é o conjunto de técnicas de análise das comunicações que visa obter por procedimentos objetivos e sistemáticos, indicadores que permitam a inferência de conhecimentos relativos à produção destas informações. Esta técnica pressupõe algumas etapas, definidas como: pré-análise; exploração do material ou codificação; tratamento dos resultados, inferência e interpretação ${ }^{(13)}$. Como resultado do processo de análise, foram elaboradas duas categorias temáticas. A primeira diz respeito à "Sintomatologia referente ao período do climatério" e a segunda "Problemas que afetam a saúde de mulheres privadas de liberdade 
durante o período do climatério".

Como limitação do processo de investigação, pode-se sinalizar que diante da necessidade de atender aos trâmites burocráticos e específicos da (SEAP-RJ), houve necessidade de restringir o tempo de coleta de dados conforme datas estipuladas pela instituição e de atender as normas vigentes de revista íntima para entrada do pesquisador a cada dia de realização de entrevistas no presídio.

\section{RESULTADOS}

Das sete mulheres que fizeram parte do estudo, a faixa etária variou entre 50 e 66 anos. Em relação à autodeclaração da cor, quatro mulheres se declararam brancas e três pardas. Quanto ao estado civil, cinco perfizeram afirmando que eram divorciada/separadas e viúva. Todas referiram ter filhos. Quanto à escolaridade, três delas declararam que eram analfabetas, sendo que uma sabia ler, mas não conseguia escrever; e uma com nível superior completo.

Em relação ao delito cometido, três disseram que estavam presas por tráfico de drogas, três por estelionato e uma por homicídio. Quanto ao tempo de reclusão, este foi bem variado, sendo o maior tempo para 67 anos e o menor cinco anos. Quanto ao tempo de cumprimento da pena, o maior tempo relatado foi de 20 anos e o menor de um ano e dois meses.

\section{Sintomatologia referente ao período do climatério}

Das sete mulheres entrevistadas, todas verbalizaram estar com a menstruação ausente. Sendo que a entrevistada M4 referiu sua última menstruação ocorreu quando tinha 27 anos e a M6 sinalizou a maior idade, ocorrendo aos 57 anos. Também foi possível identificar que todas as entrevistadas inicialmente não verbalizaram percepção de alterações relacionadas ao período do climatério. No entanto, com o decorrer das entrevistas, foram compartilhando informes significativos referentes aos vários sinais, sintomas ou problemas de saúde referidos pelas mulheres que apresentavam relação direta ou indireta com período do climatério. Vide as falas seguintes:

Sinto muito calor, e por isso procuro sempre estar perto do ventilador e me refrescar no chuveiro. (M3)

Estou apresentando: queda de cabelo, dificuldade para crescimento das unhas, dores na coluna e dor de cabeça. (M4)

Sinto dores na coluna e dor de cabeça. (M6)

Canto pra espantar os calores. (M5)

A maioria das entrevistadas relata que passa pelo período do climatério durante a prisão de maneira desconfortável. Sendo que na realidade os sinais, sintomas, ou problemas encontram-se de modo interdependente interferindo no cotidiano prisional dessas mulheres.

\section{Problemas que afetam a saúde de mulheres privadas de liberdade durante o período do climatério}

Existem alguns problemas característicos do período do climatério que também interferem no dia a dia das mulheres privadas de liberdade e se mesclam tomando destaque nas entrevistas. Como exemplo, pode-se observar nos relatos a existência de preocupação, tristeza, cansaço, padrão de sono alterado; reforçando o quanto o ambiente prisional pode interferir nos modos de vida diária, sobretudo durante o período do climatério.

Tenho muita insônia, aqui dentro. (M4)

Acordo várias vezes durante a madrugada assustada... me preocupo com minha família e isso me atrapalha a dormir. (M6)

Além desses, um dos problemas que mais aparece nas falas afetando a saúde das encarceradas é a 
hipertensão arterial. Pode ser clinicamente decorrente de fatores relacionados à faixa etária avançada ou proveniente do estilo de vida cotidiana e ainda de fatores institucionais. Vide os depoimentos seguintes:

Sou hipertensa e tenho problema de coração. (M7)

Sou hipertensa, sinto cansaço, dores de cabeça e esquecimento. (M4)

Como um fator que pode estar correlato, o aumento de peso após o confinamento aparece na fala de cinco entrevistadas. Tal questão pode ser exemplificada a partir da fala a seguir:

Aumentei $15 \mathrm{~kg}$ após confinamento, engordei muito aqui dentro. (M4)

Não me peso, mas engordei, a calça não fecha mais. (M5)

Outros problemas que aparecem de modo aproximado em algumas entrevistas e que de algum modo podem estar relacionados ao climatério são as doenças crônicas não transmissíveis (DCNT) como diabetes, hérnia de disco, artrose, asma e catarata. Como pode ser exemplificado a partir dos seguintes relatos:

Tenho enfisema pulmonar crônico, já fui ao medico, pego remédio na enfermagem quando tem, nunca fui atendida por outro profissional, queria saber por que tenho tanto suor e nunca fiz tratamento pra isso. (M7)

Só tenho problema de diabetes, hipertensão e visão. (M2)

\section{- DISCUSSÃO}

As análises foram realizadas levando-se em consideração a frequência que as variáveis foram surgindo nas entrevistas. Como pode ser visto, dentre os inúmeros sinais, sintomas e problemas ou fatores que afetam a saúde associados ao período do climatério durante o período prisional são: a sensação de calor aumentado, sudorese e preocupação formam um conjunto que se destaca nas falas das participantes.

A literatura sinaliza que esses sinais e sintomas podem apresentar-se exacerbados mediante as alterações hormonais vivenciadas pela mulher, pois as alterações hormonais sofridas pelas mulheres podem dialogar com a presença de tristeza profunda e alterações frequentes do humor ${ }^{(14)}$.

Essas alterações de humor podem estar relacionadas aos processos de sofrimento psíquico. Das sete entrevistadas, duas disseram que já utilizaram o serviço de saúde mental antes da prisão, sendo que duas eram acompanhadas pelo Centro de Apoio Psicossocial (CAPS) e duas já relataram que foram internadas em hospital psiquiátrico. Mesmo sendo minoria nesse estudo, esse dado é de suma importância, pois o período do climatério exacerba possibilidade de sofrimento psíquico ${ }^{(15)}$, possibilitando desencadear transtornos psíquicos comuns nas prisões ${ }^{(10)}$.

Ainda com relação aos fatores que afetam a saúde desse grupo entrevistado, também surgiram, em algumas falas, questões relacionadas à preocupação, cansaço, estresse e padrão de sono alterado, fatores que podem estar relacionados à diminuição de serotonina e ao hipoestrogenismo. As ondas de calor, sudorese e vaginite atrófica são os únicos sintomas caracterizados como decorrentes do hipoestrogenismo(15).

Além destes, também foram identificados durante o período do climatério as ondas de calor ou "fogachos", sudorese e sono agitado. O "nervosismo" e os "fogachos" encontram-se presentes entre a maioria das sintomatologias proferidas pelas mulheres durante período do climatério, sendo que algumas destas queixas estão inter-relacionadas ${ }^{(15)}$. Nessa perspectiva, os resultados estão de acordo com a literatura consultada, em que $92,07 \%$ das mulheres experimentaram pelo menos um dos sintomas, com maior frequência de fogachos, irritabilidade e esquecimento ${ }^{(16)}$.

Vale apontar que a mulher que se encontra em situação de prisão tem limitadas suas ações e o direito de livre acesso nos espaços institucionais. Assim, mediante dificuldade de controlar as próprias 
decisões, essa mulher apresenta não só alterações relacionadas aos aspectos biológicos, como também às questões emocionais, tornando-a vulnerável para a presença de outras morbidades.

Outros sinais e sintomas citados em menor frequência pelas entrevistadas foram esquecimento, baixa autoestima, melancolia, depressão, mastalgia, alteração da libido, incontinência urinária e ressecamento da vagina aparecem sendo citados apenas uma vez. Dentre esses, os primeiros podem caracterizar alterações cotidianas que de algum modo dialogam com situações específicas relacionadas à saúde mental; sendo o ressecamento e/ou atrofia da vagina o sinal que tem maior relação com período do climatério.

Dessa forma, os sintomas relacionados à depressão, por exemplo, foi um dos que mais referiram as mulheres entrevistadas, confirmando-se que a transição do climatério tem grande impacto na vida ${ }^{(16)}$. Quanto ao ressecamento da vagina, a secura vaginal, que ocorre em maior frequência de quatro a seis anos após a menopausa, está diretamente relacionada ao estado de hipoestrogenismo ${ }^{(15)}$.

No que se refere ao sistema prisional, pode-se inferir que as mulheres apresentam dificuldades para manter níveis pressóricos normais, pois, além de hábitos individuais referentes à alimentação ,existe dificuldade institucional em manter a dieta saudável/balanceada, bem como a atividade física frequente e direcionada.

As atividades físicas e de lazer vem ganhando cada vez mais destaque para as pessoas privadas de liberdade ${ }^{(17)}$. No entanto, a prática de atividade física ainda são abordagens pouco existentes no sistema prisional ${ }^{(18)}$.

Atrelado a essas questões, observa-se que durante o período do climatério também pode ocorrer um aumento de peso relativo e concentrado no abdome. Entendendo que durante a prisão existe a possibilidade de dificuldade relacionada à alimentação e sedentarismo ${ }^{(18)}$.

Os sintomas de dispneia, lombalgia/cervicalgia e insônia também aparecem como estados mórbidos prevalentes no grupo. Dentre os inúmeros fatores que podem afetar a saúde de mulheres privadas de liberdade, o padrão de sono e repouso alterado aparece na fala de seis entrevistadas, sendo referido como presença de "insônia". Sabe-se que esta questão, caracterizada pela dificuldade de dormir, piora durante o climatério afetando a saúde desse grupo específico de mulheres ${ }^{(19)}$.

Dessa forma, a insônia é frequentemente atribuída às ondas de calor, mas também pode estar associada aos sintomas psicológicos e/ou ambientais ${ }^{(15)}$. No sistema prisional, os horários de sono e vigília são impostos pela rotina institucional e comumente diferenciados dos hábitos pessoais de cada mulher. Assim, principalmente em situações adversas como as instituições prisionais, a insônia referida por essas mulheres pode estar agravada.

No entanto, como são problemas sinalizados em outros períodos da vida, há necessidade de maiores estudos apresentando correlações com o climatério. Mesmo assim, vale destacar que mediante os múltiplos fatores estruturais/institucionais que caracterizam as prisões, a mulher que ingressa no sistema prisional raramente sai do mesmo sem ser acometida por alguma doença ${ }^{(18)}$.

\section{CONSIDERAÇÕES FINAIS}

As mulheres privadas de liberdade, ao passarem pelo período do climatério, lidam com os sintomas e os problemas que afetam a saúde no dia a dia de confinamento, convivendo com barreiras diárias e imposições institucionais. Ao serem questionadas sobre como vivenciam esse período, pode-se observar como esse grupo vulnerável feminino reconhece as dificuldades vivenciadas durante o climatério, sobretudo quando sobrepostas a situações próprias da privação de liberdade. A maioria das entrevistadas referiu passar pelo período do climatério de maneira desconfortável e/ou naturalizada.

Torna-se interessante que a enfermeira e equipe multiprofissional de saúde, a partir da identificação de questões referentes à fase da vida dessas mulheres, estejam susceptíveis ao exercício de realizar acolhimento, provocar ações de autocuidado, prevenir agravos, tratar doenças e promover saúde; a fim de minimizar situações desconfortáveis e contribuir para melhorar condições de vida durante o tempo de encarceramento. 
Assim, o período do climatério, sobretudo em situações específicas como a prisão, deve ser mais explorado pelas políticas públicas de saúde contribuindo para melhoria das condições de vida desse grupo específico de mulheres.

\section{- REFERÊNCIAS}

1. Küchemann BA. Envelhecimento populacional, cuidado e cidadania:velhos dilemas e novos desafios. Soc. Estado. [Internet] 2012;27(1) [acesso em 12 ago 2016]. Disponível: http://dx.doi.org/10.1590/S0102-69922012000100010.

2. Ministério do Planejamento (MP). Instituto Brasileiro de Geografia e Estatístico (IBGE). Síntese de Indicadores Sociais, uma análise das condições de vida da população brasileira. Rio de Janeiro: MP; IBGE; 2013.

3. de Lorenzi DRS, Catan LB, Moreira K, Ártico GR. Assistência à mulher climatérica: novos paradigmas. Rev. bras. enferm. [Internet] 2009;62(2) [acesso em 12 ago 2016]. Disponível: http://dx.doi.org/10.1590/S003471672009000200019 .

4. Maron L, Leal A, Bandeira D, Macedo PS, Garcia SS, da Silva EB. A Assistência às mulheres no climatério: um estudo bibliográfico. Revista Contexto \& Saúde. [Internet] 2011;11(20) [acesso em 12 ago 2016]. Disponível: https://www.revistas.unijui.edu.br/index.php/contextoesaude/article/view/1576/1331.

5. Fonseca FM, dos Santos FF, da Costa FM, Santos JAD, Carneiro JA. Climatério: influência na sexualidade feminina. Rev. Univ. Vale Rio Verde. [Internet] 2015;13(2) [acesso em 12 ago 2016]. Disponível: http://dx.doi. org/10.5892/ruvrd.v13i1.2382.

6. Ministério da Saúde (BR). Secretária de Atenção à Saúde. Manual de Atenção à Mulher no Climatério/ Menopausa. Brasília; Secretária de Atenção à Saúde; 2008.

7. Ministério da Saúde (BR). Portaria Interministerial n.1 de 2 de janeiro de 2014. Institui Política Nacional de Atenção Integral à Saúde das Pessoas Privadas de Liberdade no Sistema Prisional. Gabinete do ministro, [Internet] 02 jan 2014 [acesso em 10 jan 2016]. Disponível:http://www.lex.com.br/legis_25201439_portaria_interministerial_n_1_ de_2_de_janeiro_de_2014.aspx.

8. Ministério da Justiça (BR). Portaria Interministerial $n^{\circ} 210$ de 16 de janeiro de 2014. Institui Política Nacional de Atenção às Mulheres em Situação de Privação de Liberdade e Egressas do Sistema Prisional, e dá outras providências. Gabinete do ministro, [Internet] 16 de jan 2014 [acesso em 10 jan 2016]. Disponível: http://www.lex. com.br/legis_25232895_portaria_interministerial_n_210_de_16_de_janeiro_de_2014.aspx.

9. Almeida CA, da Cruz SS. Sistema Penitenciário Brasileiro: uma análise da penitenciária industrial regional do Cariri - PIRC. Revista Direito \& Dialogicidade. [Internet] 2014;5(2) [acesso em 10 jan 2016]. Disponível: http:// periodicos.urca.br/ojs/index.php/DirDialog/article/view/878/781.

10. Damas FB, de Oliveira WF. A saúde mental nas prisões de Santa Catarina, Brasil. Cadernos Brasileiros de Saúde Mental. [Internet] 2013;5(13) [acesso em 10 jan 2016]. Disponível: http://incubadora.periodicos.ufsc.br/ index.php/cbsm/article/view/1758/3184.

11. Minayo MCS. O Desafio do Conhecimento: pesquisa qualitativa em Saúde. 14ª ed. São Paulo: Hucitec; 2014.

12. Ministério da Saúde (BR). Conselho Nacional de Saúde. Diretrizes e normas regulamentadoras de pesquisa envolvendo seres humanos. Resolução n. 466, de 12 de dezembro de 2012. Brasília; 2012.

13. Bardin L. Análise de Conteúdo. 6ª Ed. Lisboa; Edições 70; 2011.

14. Monteiro MLS. Como vai a senhora? Reflexões sobre as perdas e angústias da mulher madura. IGT na Rede. [Internet] 2010;7(13) [acesso em 10 jan 2016]. Disponível: http://biblat.unam.mx/pt/revista/igt-na-rede/articulo/ como-vai-a-senhora-reflexoes-sobre-as-perdas-e-angustias-da-mulher-madura.

15. Pedro AO, Pinto-Neto AM, Costa-Paiva LHS, Osis MJD, Hardy EE. Síndrome do climatério: inquérito populacional domiciliar em Campinas, SP. Rev. Saúde Publica. [Internet] 2003;37(6) [acesso em 10 jan 2016]. Disponível: http://dx.doi.org/10.1590/S0034-89102003000600008.

16. Pitombeira R, Lima FET, Magalhães FJ, Custódio IL, de Oliveira SKP. Sintomatologia e modificações no cotidiano 
das mulheres no período do climatério. Cogitare Enferm. [Internet] 2011;6(3) [acesso em 10 jan 2016]. Disponível: http://dx.doi.org/10.5380/ce.v16i3.20913.

17. Moraes AM, de Moraes BM, Ramos VM. A prática da atividade física no presídio: o que pensam os apenados? Caderno de Educação Física e Esporte. [Internet] 2014;12(1) [acesso em 10 jan 2016]. Disponível: http://e-revista. unioeste.br/index.php/cadernoedfisica/article/view/9794/8797.

18. Silva EM. A mulher detenta, a sua saúde sexual e sua sexualidade: revisão sistemática da literatura brasileira sobre a atuação da enfermagem neste processo [dissertação]. São Paulo (SP): Universidade de São Paulo; 2013.

19. Reis CB, Bernardes EB. O que acontece atrás das grades: estratégias de prevenção desenvolvidas nas delegacias civis contra HIV/AIDS e outras doenças sexualmente transmissíveis. Cienc. Saúde Colet. [Internet] 2011;16(7) [acesso em 10 jan 2016]. Disponível: http://dx.doi.org/10.1590/S1413-81232011000800032. 